\title{
RIPPLY2 is differentially expressed in brain metastatic human breast cancer.
}

Shahan Mamoor, MS ${ }^{1}$

$3 \quad$ 'shahanmamoor@gmail.com

East Islip, NY USA

Metastasis to the brain is a clinical problem in patients with breast cancer ${ }^{1-3}$. We mined published microarray data ${ }^{4,5}$ to compare primary and metastatic tumor transcriptomes for the discovery of genes associated with brain metastasis in humans with metastatic breast cancer. We found that ripply transcriptional repressor 2, encoded by RIPPLY2, was among the genes whose expression was most different in the brain metastases of patients with metastatic breast cancer as compared to primary tumors of the breast. RIPPLY2 mRNA was present at decreased quantities in brain metastatic tissues as compared to primary tumors of the breast. Importantly, expression of RIPPLY2 in primary tumors was significantly correlated with patient recurrence-free survival. Modulation of RIPPLY2 expression may be relevant to the biology by which tumor cells metastasize from the breast to the brain in humans with metastatic breast cancer.

Keywords: breast cancer, metastasis, brain metastases, central nervous system metastases, ripply transcriptional repressor 2, RIPPLY2, systems biology of breast cancer, targeted therapeutics in breast cancer. 
One report described a 34\% incidence of central nervous system metastases in patients treated with trastuzumab for breast cancer ${ }^{2}$. More recently, the NEfERT-T clinical trial ${ }^{6}$ which compared administration of either neratinib or trastuzumab in conjunction with paclitaxel demonstrated that in a randomized, controlled setting, in breast cancer patients treated with neratinib, not only was the incidence of central nervous system recurrence significantly lower, the time to central nervous system metastasis was significantly delayed as compared to patients administered trastuzumab ${ }^{6}$. The alarmingly high rate of central nervous system metastasis described, as well as data, both anecdotal ${ }^{2}$ and from a randomized, controlled setting ${ }^{6}$ illustrating that treatment with trastuzumab may be associated with these events demands an enhanced understanding of the transcriptional makeup of brain metastatic tissues to support identification of therapeutic targets, whether they are treatment related or not. We performed a global comparative analysis of primary and metastatic tumors in patients with brain metastatic breast cancer ${ }^{4,5}$. We discovered differential and decreased expression of the gene encoding ripply transcriptional repressor 2, RIPPLY2, in brain metastatic tissues of patients with metastatic breast cancer.

\section{Methods}

We used datasets GSE10893 ${ }^{4}$ and GSE100534 ${ }^{5}$ for this global differential gene expression analysis of brain metastatic breast cancer in conjunction with GEO2R. GSE10893 was generated using Agilent-011521 Human 1A Microarray G4110A technology with $n=11$ primary breast tumors and $n=4$ brain metastases from patients with breast cancer; analysis was performed using platform GPL885. GSE100534 was generated using Affymetrix Human Gene 1.0 ST Array with $n=16$ primary breast tumors and $n=3$ brain metastases from patients metastatic breast cancer; analysis was performed using platform GPL6244. The Benjamini and Hochberg method of $p$-value adjustment was used for ranking of differential expression but raw $p$-values were used to assess statistical significance of global differential expression. Log-transformation of data was auto-detected, and the NCBI generated category of platform annotation was used. A statistical test was performed to evaluate whether RIPPLY2 gene expression was significantly different between primary tumors of the breast and brain metastases in humans with breast cancer using a two-tailed t-test. For Kaplan-Meier survival analysis, we used the Kaplan-Meier plotter online tool $^{7}$ for correlation of RIPPLY2 mRNA expression levels with recurrence-free survival in $n=1764$ breast cancer patients.

\section{$\underline{\text { Results }}$}

We performed global comparative transcriptome analysis of metastatic tumor tissues of patients with metastatic breast cancer using published microarray data ${ }^{4,5}$ to describe the transcriptional landscape of brain metastasis in human breast cancer in an unbiased fashion and for the discovery of novel therapeutic targets.

RIPPLY2 is differentially expressed in the brain metastases of patients with brain metastatic breast cancer.

Through blind, systems-level analysis of published microarray data ${ }^{4}$, we identified the ripply transcriptional repressor 2, encoded by RIPPLY2, as a differentially expressed gene in the breast metastatic tissues of humans with breast cancer (Table 1). When sorting each of the genes expressed in brain metastases based on significance of difference as compared to primary tumors of the breast in patients with breast cancer, RIPPLY2 ranked 215 out of 17418 total transcripts (Chart 1), equating to 98.8\% differential expression. Differential expression of RIPPLY2 in the brain metastases of patients with metastatic breast cancer was statistically significant (Chart $1 ; p=6.05 \mathrm{e}-04)$. 
To attempt to validate transcriptome-wide differential expression of RIPPLY2 in brain metastatic tissues of patients with metastatic breast cancer, we queried a second microarray dataset ${ }^{5}$, here comparing primary tumors from patients with breast cancer to brain metastases. Again, we identified RIPPLY2 as a differentially expressed gene in human brain metastatic breast cancer (Chart 2) When sorting each of the genes expressed in brain metastases based on significance of difference as compared to normal breast tissues, RIPPLY2 ranked 9626 out of 41093 total transcripts (Chart 2), equating to $76.6 \%$ differential expression. Differential expression of RIPPLY2 in the brain metastases of patients with metastatic breast cancer was statistically significant (Chart $2 ; p=7.91 \mathrm{e}-02$ ). Thus, differential expression of RIPPLY2, transcriptome-wide, in the brain metastases of women with metastatic breast cancer was conserved across two independent microarray datasets. This suggests that RIPPLY2 differential expression in human brain metastatic breast cancer is a bona fide biological process.

RIPPLY2 is expressed at lower levels in the brain metastases of patients with metastatic breast cancer.

We obtained exact mRNA expression levels for RIPPLY2, in primary tumors of the breast and in brain metastasis of patients with brain metastatic breast cancer to determine direction and statistical significance of change in RIPPLY2 expression in brain metastatic tissues. We observed significantly lower expression of RIPPLY2 in the brain metastases of patients with breast cancer as compared to primary tumors of the breast: RIPPLY2 was expressed at $0.27 \pm 0.49$ arbitrary units (AU) in primary tumors of the breast, while it was expressed at $-0.85 \pm 0.32 \mathrm{AU}$ in brain metastatic tissues. The difference in RIPPLY2 mRNA levels between primary tumors of the breast and brain metastatic tissues was statistically significant (Figure $1 ; p=0.001038$ ).

\section{RIPPLY2 expression is significantly correlated with survival outcomes in human breast cancer.}

We performed Kaplan-Meier survival analysis ${ }^{7}$ in $n=1764$ breast cancer patients in total, to evaluate whether RIPPLY2 tumor expression was correlated with survival outcomes in breast cancer. We observed a statistically significant correlation between primary tumor expression of RIPPLY2 and recurrence-free survival (RFS) in patients with breast cancer, in the lower survival quartile (Figure 2). Patients whose primary tumors expressed low levels of RIPPLY2 possessed median RFS of 30 months, while patients whose tumors expressed high levels of RIPPLY2 possessed median RFS of 38 months. significant (Figure 2, Chart 3; logrank $p$-value: 0.023; hazard ratio: 0.83 (0.7-0.97)).

Thus, by mining published microarray data ${ }^{4,5}$ in an unbiased and systematic fashion, we identified ripply transcriptional repressor 2, encoded by RIPPLY2, as among the genes whose expression was most different, transcriptome-wide, in the brain metastases of patients with breast cancer when compared to primary tumors of the breast; we observed significantly decreased expression of RIPPLY2 in brain metastases as compared to primary tumors of the breast. Further, we found a significant correlation between RIPPLY2 expression and patient survival outcomes, as recurrence-free survival was significantly greater in patients whose primary tumors expressed higher levels of RIPPLY2 as compared to patients whose primary tumors expressed lower levels of RIPPLY2.

\section{Discussion}

We provided evidence here that ripply transcriptional repressor 2, encoded by RIPPLY2, is among the genes whose expression is most different in the brain metastases of patients with brain metastatic breast cancer, that RIPPLY 2 mRNA is present at significantly decreased quantities in brain metastatic tissues as compared to primary tumors of the breast, and that primary tumor RIPPLY2 expression is 


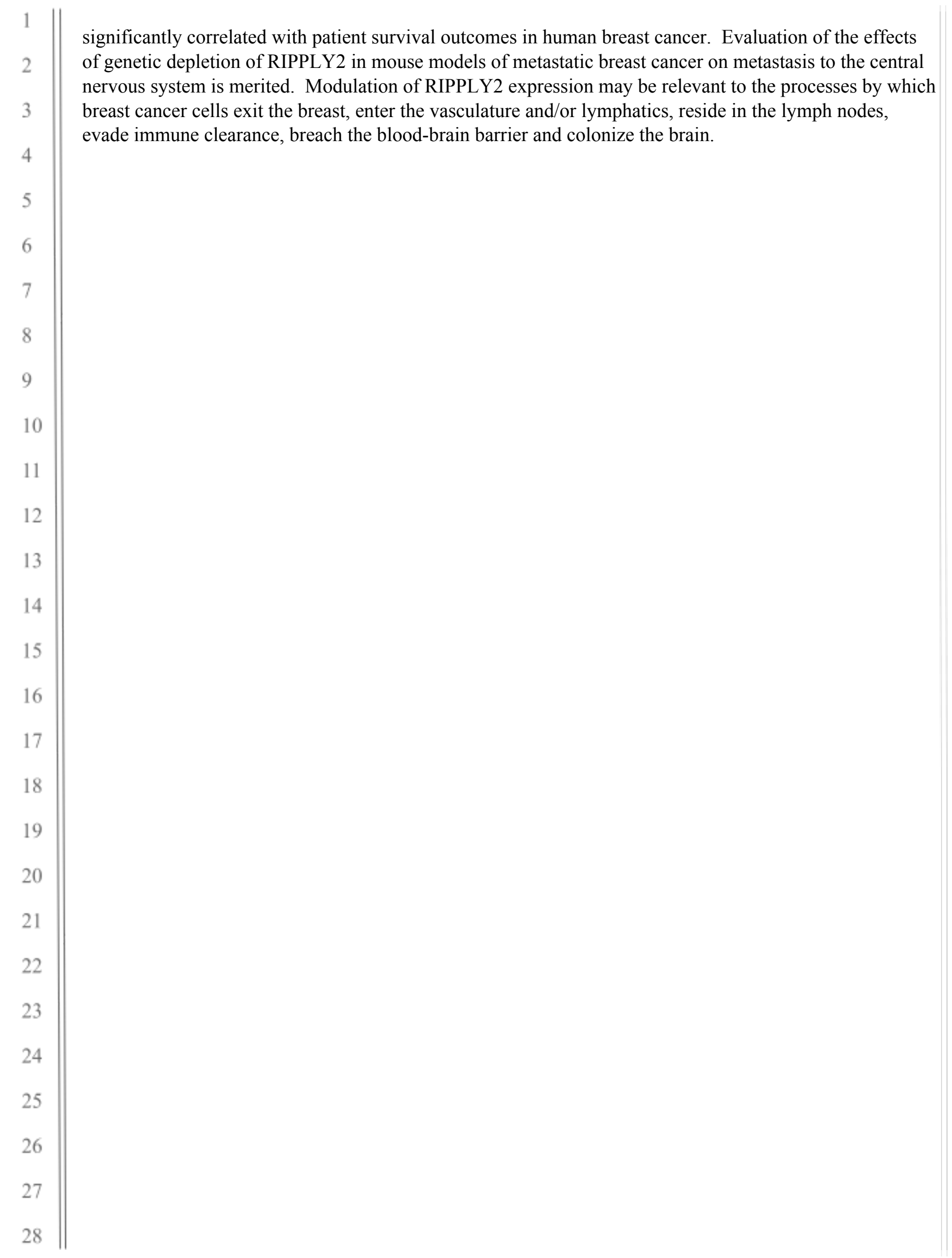




\section{References}

1. Lin, N.U., Amiri-Kordestani, L., Palmieri, D., Liewehr, D.J. and Steeg, P.S., 2013. CNS metastases in breast cancer: old challenge, new frontiers.

2. Bendell, J.C., Domchek, S.M., Burstein, H.J., Harris, L., Younger, J., Kuter, I., Bunnell, C., Rue, M., Gelman, R. and Winer, E., 2003. Central nervous system metastases in women who receive trastuzumab-based therapy for metastatic breast carcinoma. Cancer, 97(12), pp.2972-2977.

3. Tsukada, Y., Fouad, A., Pickren, J.W. and Lane, W.W., 1983. Central nervous system metastasis from breast carcinoma autopsy study. Cancer, 52(12), pp.2349-2354.

4. Weigman, V.J., Chao, H.H., Shabalin, A.A., He, X., Parker, J.S., Nordgard, S.H., Grushko, T., Huo, D., Nwachukwu, C., Nobel, A. and Kristensen, V.N., 2012. Basal-like Breast cancer DNA copy number cancer research and treatment, 133(3), pp.865-880.

5. Schulten, H.J., Bangash, M., Karim, S., Dallol, A., Hussein, D., Merdad, A., Al-Thoubaity, F.K., Al-Maghrabi, J., Jamal, A., Al-Ghamdi, F. and Choudhry, H., 2017. Comprehensive molecular biomarker identification in breast cancer brain metastases. Journal of translational medicine, 15(1), p.269.

6. Awada, A., Colomer, R., Inoue, K., Bondarenko, I., Badwe, R.A., Demetriou, G., Lee, S.C., Mehta, A.O., Kim, S.B., Bachelot, T. and Goswami, C., 2016. Neratinib plus paclitaxel vs trastuzumab plus paclitaxel in previously untreated metastatic ERBB2-positive breast cancer: the NEfERT-T randomized clinical trial. JAMA oncology, 2(12), pp.1557-1564.

7. Györffy, B., Lanczky, A., Eklund, A.C., Denkert, C., Budczies, J., Li, Q. and Szallasi, Z., 2010. An online survival analysis tool to rapidly assess the effect of 22,277 genes on breast cancer prognosis using microarray data of 1,809 patients. Breast cancer research and treatment, 123(3), pp.725-731. 


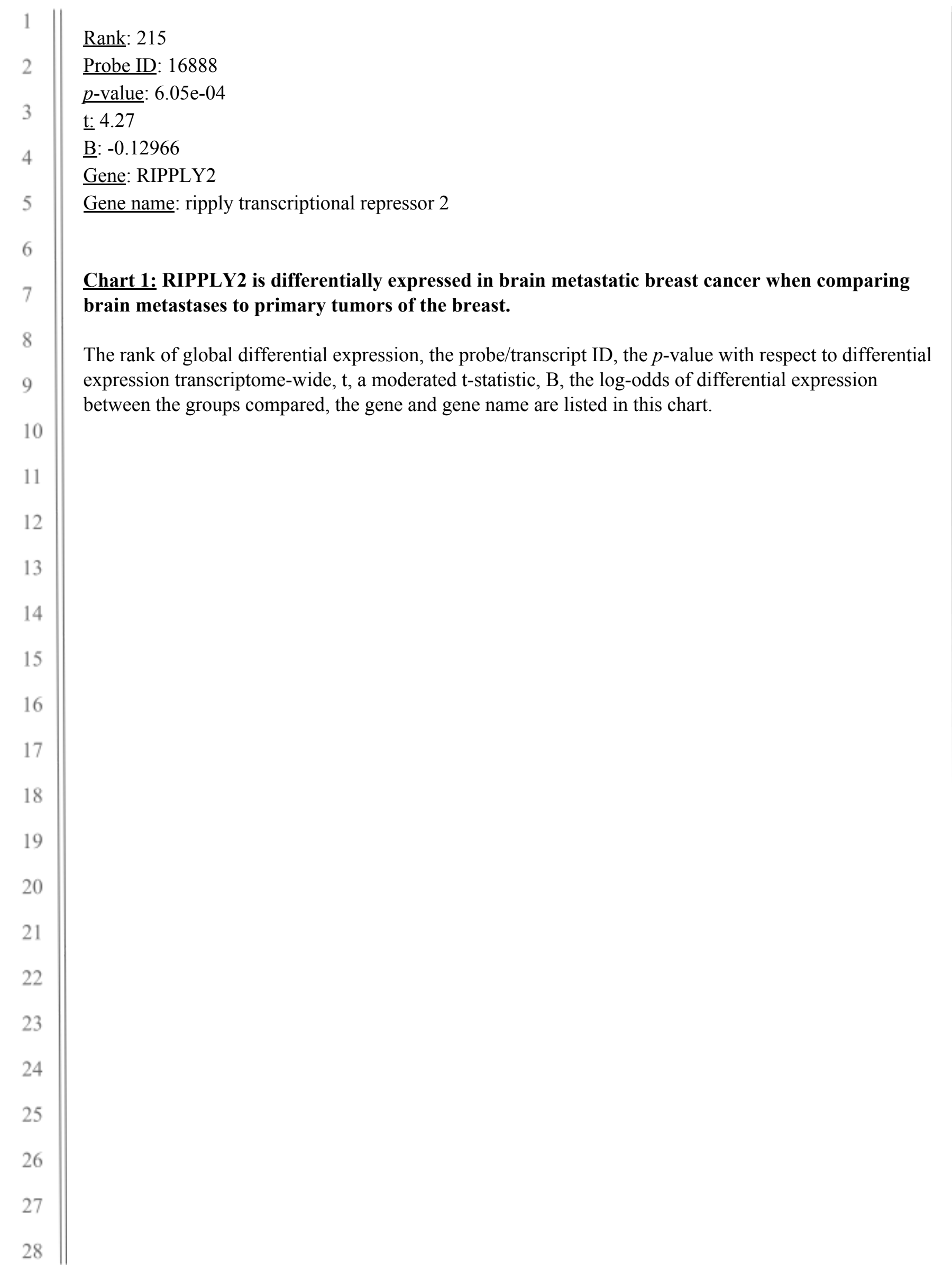




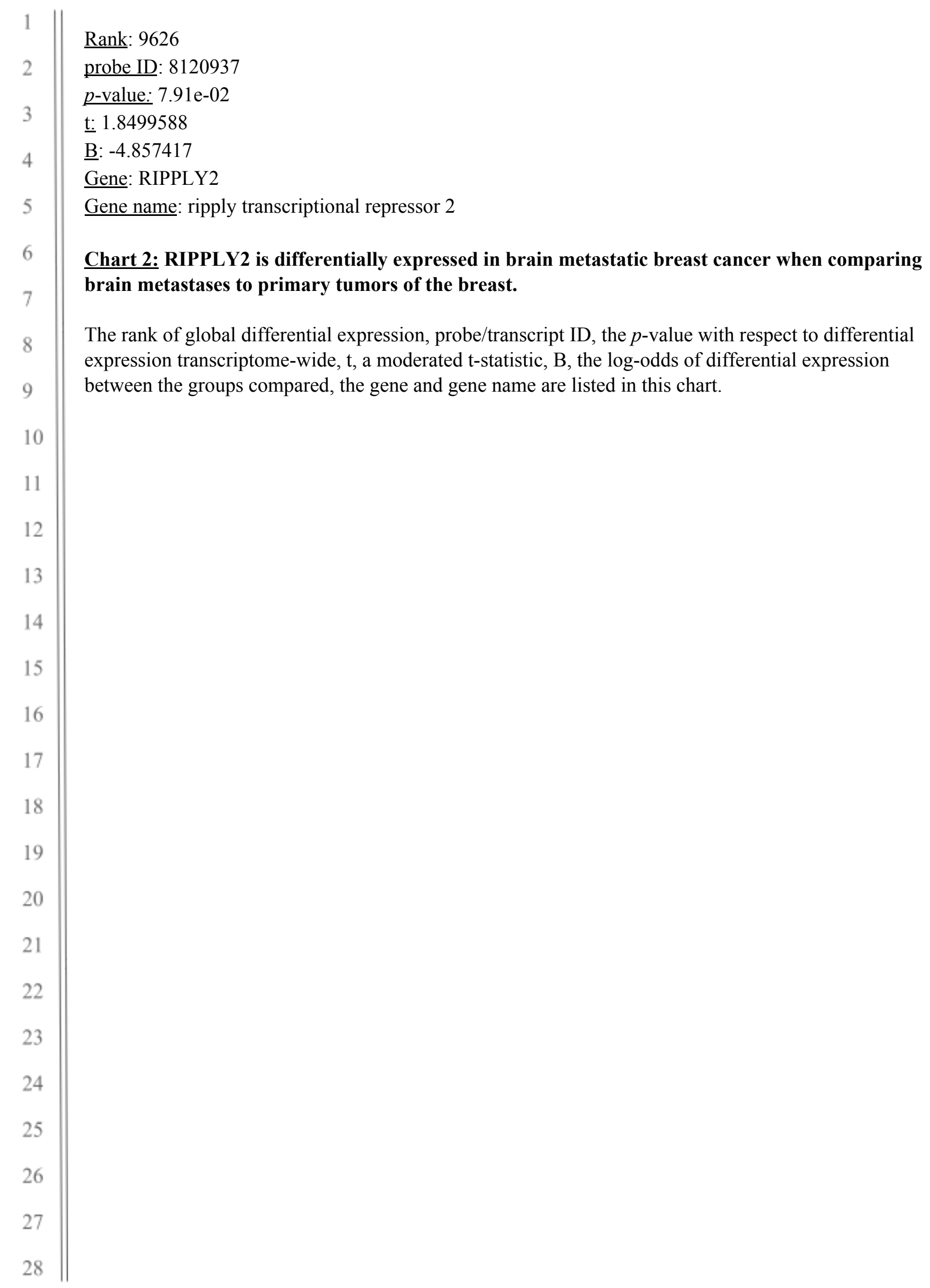




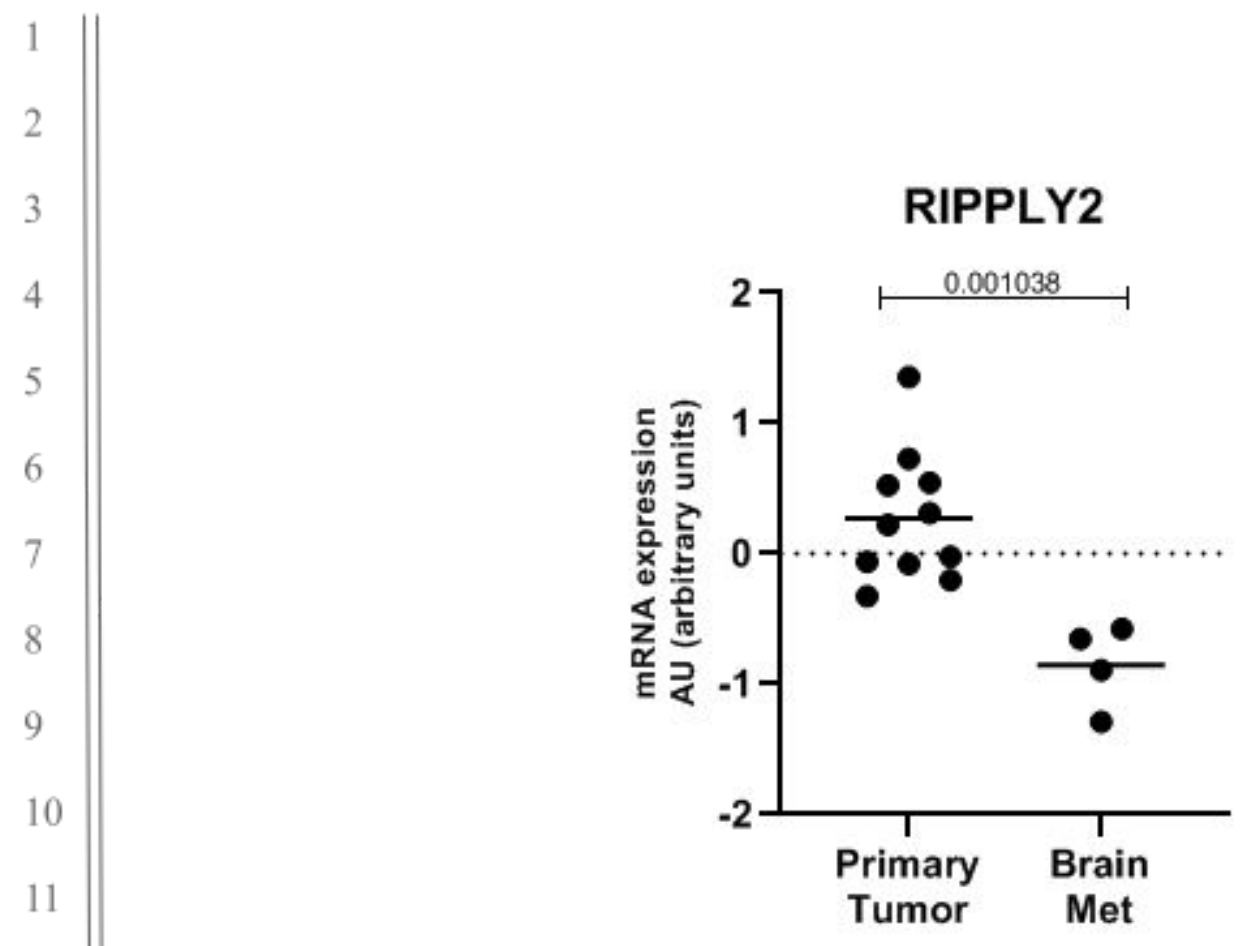

Figure 1: RIPPLY2 is expressed at significantly lower levels in the brain metastases of patients with metastatic breast cancer when compared to primary tumors of the breast.

The mRNA expression level of RIPPLY2 in primary tumors of the breast (left) and in brain metastases of women with metastatic breast cancer (right) is graphically depicted; the result of a statistical test evaluating significance of difference in RIPPLY2 expression between primary tumors of the breast and brain metastases, a $p$-value, is $p=0.001038$. 


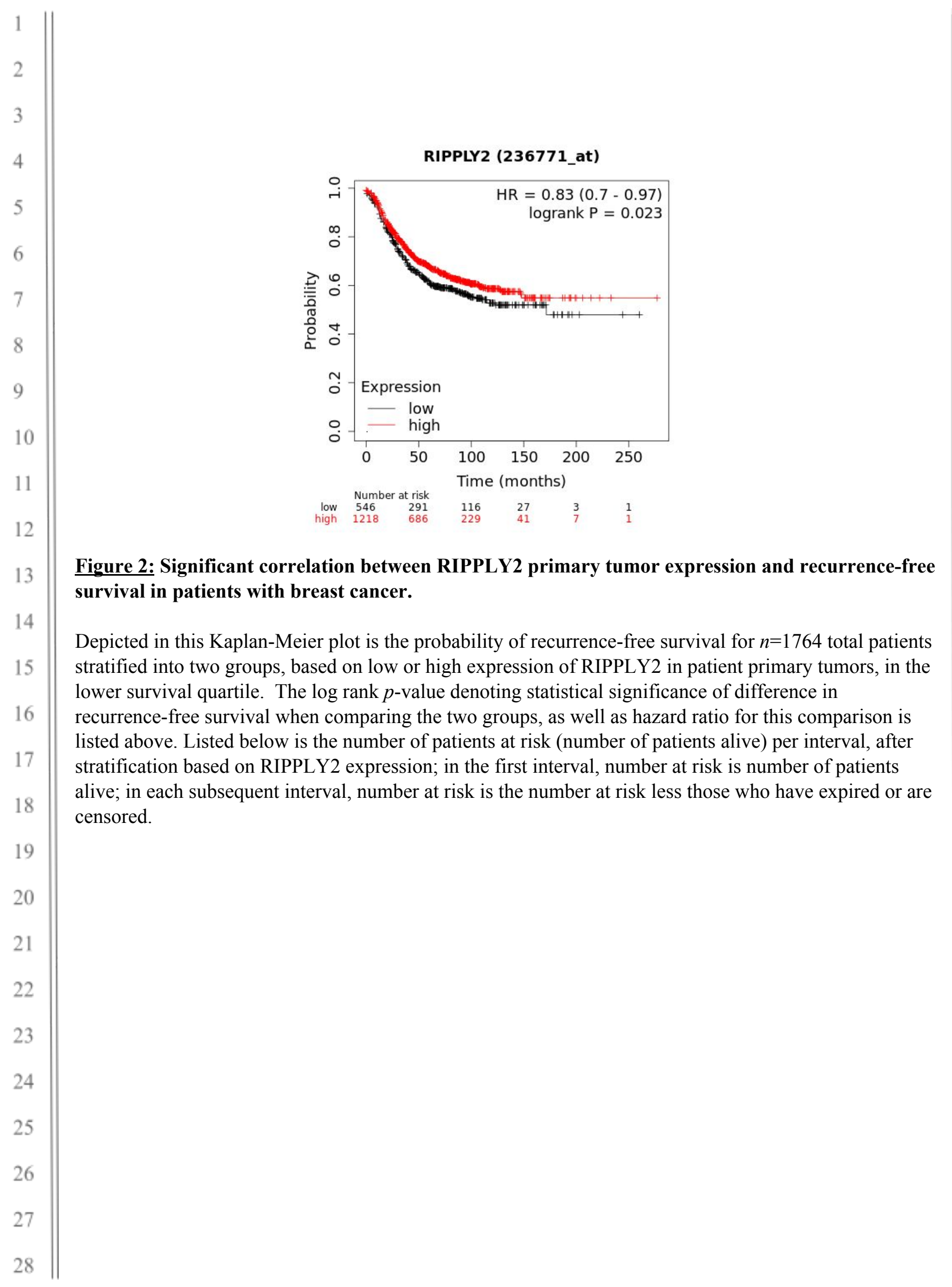




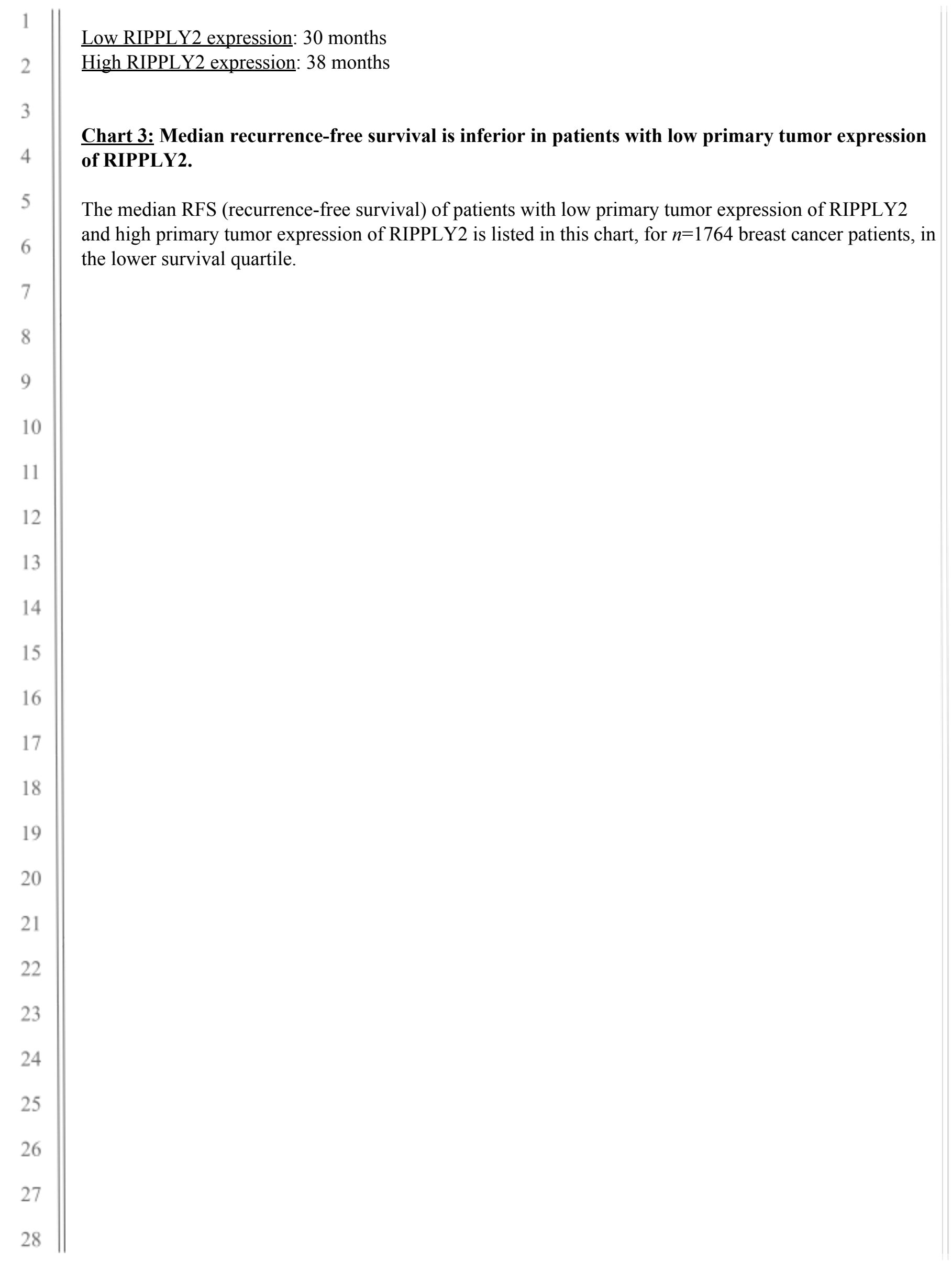

